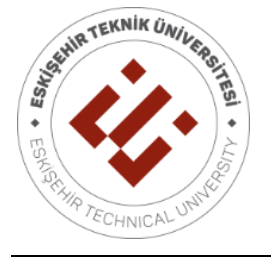

ESKIŞEHIR TECHNICAL UNIVERSITY JOURNAL OF SCIENCE AND TECHNOLOGY A- APPLIED SCIENCES AND ENGINEERING

\title{
THE OVERVIEW OF METHOD OF BALANCED RESOURSE RESERVATION AND MULTICAST ROUTING IN TELECOMMUNICATION NETWORK
}

\author{
Amal MERSNI ${ }^{1}$, Olena NEVZOROVA ${ }^{2, *}$, Yelyzaveta TOLSTYKH ${ }^{3}$ \\ 1,2 Infocommunication engeeniring Department, Infocommunication Faculty, \\ Kharkiv National University of Radio Electronic, Kharkiv, Ukraine \\ ${ }^{3}$ Department of Media Engıneerıng and information radioelectronıc systems, Faculty of Information Radıo Technologies and \\ Technical Information Security, Kharkiv National University of Radio Electronic, Kharkiv, Ukraine
}

\begin{abstract}
The overview of a mathematical model for multicast routing supporting shared explicit reservation of link resource was presented, which is introduced by linear expressions that responsible for ensuring the connectivity of the calculated multicast routes, as well as the absence of loops in them. The novelty of the model consists in introducing conditions for preventing congestion of communication links when implementing a shared explicit reservation, in which a link resource is allocated to several flows simultaneously, but the list of these flows is strictly defined. The task of routing multicast flows supporting shared explicit reservation of link resource is formulated as an optimization problem of mixed integer linear programming. The use of the proposed model makes it possible to exploit the available link resource more efficiently (on average, from 15 to $25 \%$ ) by ensuring the consistency of solutions for multicast routing tasks and organizing of the shared explicit reservation.
\end{abstract}

Keywords: Multicast routing, Resource reservation, Network, Flow-based model

\section{INTRODUCTION}

The increase in the level of Quality of Service (QoS) in modern infocommunication networks is largely due to an increase in the level of consistency in the solutions of individual traffic management tasks. The most important tasks are routing and network resources reservation. In IP networks, various technological means are responsible for solving these network problems: the appropriate routing protocols are used to calculate routes, for example, RIP, OSPF, IGRP/EIGRP [1], and the RSVP protocol (Resource ReSerVation Protocol) is used to reserve link and buffer resources [2]. At the 0same time, the RSVP protocol functions after the end of the routing protocols, which negatively affects the efficiency of traffic management and the level of QoS, because the routing protocol can calculate the route along which a given amount of network resource may not be. This will lead to the initialization of both re-solving routing problems and the implementation of network resource reservation, which will negatively affect such important temporal QoS indicators as the average delay and packet jitter. Achieving the maximum level of consistency in solving routing and reservation tasks is possible only with a unified mathematical model that describes these processes in their relationship.

\section{THE OVERVIEW OF METHOD OF BALANCED SHARED EXPLICIT RESERVATION FOR MULTICAST ROUTING IN TELECOMMUNICATION NETWORK}

In the course of modeling multicast routing processes, the structure of the TCS is represented by a directed weighted graph, as shown in [2-4], where the nodes of the graph are network routers, and the communication channels connecting these routers are edges. Also in these works it was shown that the result of solving the routing problem of multicast flows is the set of Boolean variables, each of which numerically determines the fraction of the intensity of the flow flowing in the communication channels. During the study, the mathematical model of streaming multicast routing was chosen as the basis. Within

*Corresponding Author: olena.nevzorova@ nure.ua

Received:11.10.2019 Published: 16.12.2019 
the framework of this model, it is necessary to ensure the fulfillment of the conditions for preserving the flow, the conditions for preventing overload of communication channels, and also to ensure the prevention of the formation of loops in the network, similar conditions are introduced into the model to ensure that the packet will not loop in the network.

A consistent solution to the problems of routing and reservation of network resources was achieved by establishing a relationship between route variables and reservation variables. This relationship is represented by bandwidth reservation conditions in each communication channel. To prevent overloading the communication channels of the network, additional restrictions are introduced whose fulfillment ensures the reservation of only the available channel resource of the network. When formulating the optimality criterion for multicast routing solutions with support for general explicit reservation, it is important, firstly, to ensure that the technological implementation of these processes is taken into account, and secondly, to have effective methods for solving the formulated optimization problem. For this purpose, it is proposed to use in the future to compare the effectiveness of the following three criteria.

The first criterion is represented by the minimum of the linear objective function which consists of the sum where the first term describes the total metric of solving routing problems, and the second is the total conditional cost of solving the problem of general explicit reservation. The use of the second criterion is based on the calculation of route variables and reservation variables in the course of minimizing the introduced upper threshold for the reserved bandwidth of communication channels in the network as a whole, which should contribute to the balanced use of the channel resource of the network when it is reserved. The third criterion was the minimum of the complex objective function, the use of which contributes not only to a balanced reservation of the channel resource of the network as a whole, but also minimizes the use of this resource by flows of each group.

\section{CONCLUSION}

Thus, the multicast routing problem with support for the general explicit reservation of the channel resource is formulated in an optimization form, while at least one of the three objective functions listed above can act as a criterion. The novelty of the model is the introduction of a system of conditions for preventing overload of communication channels for the balanced use of network resources when organizing a general explicit reservation of the bandwidth of communication channels. study of the proposed mathematical model and method for three different objective functions was conducted. The study showed its effectiveness and adequacy. The use of the proposed calculation model ensured an improvement in the obtained results by an average of 45-65\% compared with the distributed (inconsistent) solution of the multicast routing and reservation of channel resources used in modern network protocols. In this regard, based on the results of the studies, the proposed criteria can be recommended for use, since their use is effective in terms of both balanced use of the network resource and in terms of ensuring maximum network performance in general.

\section{REFERENCES}

[1] Barreiros M, Lundqvist P. QoS-Enabled networks: Tools and foundations. John Wiley \& Sons; 2015 Dec 21.

[2] Lemeshko OV, Arous KM. The flow-based model of multicast routing. In Microwave and Telecommunication Technology (CriMiCo), 2013 23rd International Crimean Conference 2013 Sep 8 IEEE, 523-524

[3] Mersni A. Method of Balanced Shared Explicit Reservation for Multicast Routing in Network. Scholars Journal of Engineering and Technology (SJET); 2017; 5(4):164-169 